Pesq. Vet. Bras. 35(2):153-159, fevereiro 2015 DOI: $10.1590 /$ S0100-736X2015000200010

\title{
Aspectos da tomografia de coerência óptica em cães com retinopatia ${ }^{1}$
}

\author{
Angélica M.V. Safatle²*, Michelle B.P. Braga-Sá ${ }^{2}$ e Paulo S.M. Barros²
}

\begin{abstract}
Safatle A.M.V., Braga-Sá M.B.P. \& Barros P.S.M. 2015. [Aspects of optical coherence tomography in retinopathy in dogs.] Aspectos da tomografia de coerência óptica em cães com retinopatia. Pesquisa Veterinária Brasileira 35(2):153-159. Laboratório de Investigação em Oftalmologia Comparada, Faculdade de Medicina Veterinária, Universidade de São Paulo, Av. Prof. Dr. Orlando Marques de Paiva 87, São Paulo, SP 05508-270, Brazil. E-mail: angsaf@usp.br

The OCT is a noninvasive and noncontact exam capable to evaluate the retina and optic nerve. The OCT images provide information of the constitution of the retina and its structural integrity in vivo, providing high-resolution images that resemble optical microscopy. The objective of this paper was to describe and document the use of the optical coherence tomography (OCT) in dogs. It was possible differentiate the retinal layers of healthy dogs and compare them with dogs with progressive retinal atrophy which showed altered stratification and significant reduce of the layers. In cases of retinal detachment was observed separation of neurosensory retina from the retinal pigment epithelium, and the presence of intrarretinal exudates. Thus, the OCT was effective in the diagnosis of retinopathy.
\end{abstract}

INDEX TERMS: OCT, retinal disease, dogs, retinal detachment, progressive retinal atrophy.

RESUMO.- A tomografia de coerência óptica (OCT) é um exame não invasivo e de não contato que permite avaliar a retina e o nervo óptico. As imagens da OCT fornecem informações da constituição da retina e sua integridade estrutural in vivo, gerando imagens de alta resolução, que se assemelham à microscopia óptica. Objetivou-se descrever a técnica de tomografia de coerência óptica (OCT) e sua utilização em cães. Foi possível diferenciar claramente as camadas retinianas de cães hígidos e compará-las com as de cães portadores de atrofia progressiva de retina, que apresentaram perda da estratificação e diminuição significativa das camadas. No descolamento de retina (DR) foi possível observar a separação entre a retina neurossensorial e o epitélio pigmentário da retina (EPR), além da presença de exsudatos intrarretinianos. Assim, a OCT mostrou-se eficaz no diagnóstico de retinopatias.

TERMOS DE INDEXAÇÃO: OCT, doença retiniana, cães, descolamento de retina, atrofia progressiva da retina.

\section{INTRODUÇÃO}

\footnotetext{
${ }^{1}$ Recebido em 5 de fevereiro de 2014.

Aceito para publicação em 14 de agosto de 2014.

${ }^{2}$ Laboratório de Investigação em Oftalmologia Comparada, Departamento de Cirurgia, Faculdade de Medicina Veterinária e Zootecnia, Universidade de São Paulo (USP), Av. Prof. Dr. Orlando Marques de Paiva 87, São Paulo, SP 05508-270, Brasil. *Autor para correspondência: angsaf@usp.br
}

Recentemente desenvolveu-se uma nova técnica de imagem; a Tomografia de Coerência Óptica ou OCT (do inglês "Optical Coherence Tomography"), usada tanto em pesquisa quanto na prática clínica para avaliação da estrutura retiniana. É uma técnica de microscopia in vivo que permite avaliar a constituição da retina e sua integridade estrutural (Narfström \& Petersen-Jones 2007).

A OCT ampliou as possibilidades de diagnóstico por imagem em oftalmologia nos últimos anos, por propiciar maior confiabilidade ao diagnóstico e ao tratamento das doenças oculares (Malerbi et al. 2010). Trata-se de um exame não invasivo, de alta resolução e não contato (não há contato do aparelho com o olho), que utiliza luz semelhante ao infravermelho em um sistema de interferometria, que produz imagens seccionais da retina (Schuman 1997, Kloizman et al. 1998).

0 mecanismo de operação da OCT é semelhante ao da ultrassonografia (US) no modo B, com exceção da utilização de luz ao invés de onda sonora; e pelas imagens produzidas terem resolução aproximadamente 10 vezes maior que o US (Malerbi et al. 2010). A luz, ao atingir uma determinada interface ou estrutura anatômica, pode ser reabsorvida, transmitida ou refletida. A luz refletida pelos tecidos é captada pelo aparelho e a informação é processada por um sistema computadorizado, gerando uma imagem (Saraiva 
et al. 2010).

A OCT permite estudar a retina em perfil, com resolução microscópica, enriquecendo o estudo de várias doenças retinianas, auxiliando no diagnóstico e na monitoração de várias afecções oculares, principalmente das maculopatias no homem, tais como: edema macular presente no edema cistoide de mácula, na retinopatia diabética, no buraco macular e na degeneração macular relacionada à idade (DMRI) (Guedes \& Yamane 2003, Gouveia et al. 2006, Bordon et al. 2008, Song et al. 2010).

Estruturas que geram alta refletividade na OCT podem ser localizadas na superfície da retina, como membranas epirretinianas, hemorragias superficiais e exsudatos algodonosos; podem estar dentro da retina, como hemorragias intrarretinianas, exsudatos endurecidos e fibrose intrarretiniana; e podem ser profundas, como hiperplasia do EPR, drusas, membranas e cicatrizes sub-retinianas. Estruturas que geram baixa refletividade são, na maior parte das vezes, espaços cistoides, ou seja, áreas que contêm líquido seroso. A distinção entre sangue, fluido seroso e exsudação pode ser feita com base nas diferenças de refletividade, que são proporcionais à concentração de partículas em uma determinada coleção de líquido (Huang et al. 1991, Thomas \& Duguid 2004, Malerbi et al. 2010). Diminuição da refletividade também pode ser causada por opacificação de meios, como acontece na catarata, no leucoma e na hemorragia vítrea, pois a luz utilizada na OCT não será capaz de propagar, resultando em diminuição da reflexão em todas as camadas da retina (Fisher et al. 2009, Allemann et al. 2010). Assim sendo, a OCT indica propriedades ópticas dos tecidos e não suas características morfológicas, não sendo possível tirar conclusões histológicas ou histopatológicas baseadas apenas nas imagens da OCT (Saraiva et al. 2010).

Ao se efetuar o exame de OCT é necessário considerar os seguintes aspectos: localização, forma, refletividade e relação histológica da estrutura. É importante evidenciar que estruturas não diretamente estudadas podem afetar o sinal luminoso refletido (opacidade de meios), assim como o alinhamento inadequado do aparelho ou uma pupila pequena podem causar atenuação do sinal gerado e interpretação diagnóstica equivocada (Saraiva et al. 2010).

A OCT é um exame recente também na oftalmologia veterinária e seu uso não está difundido na maioria dos centros veterinários. Contudo, como é um exame não invasivo, de não contato e alta resolução, tem importância ímpar no diagnóstico de doenças retinianas e do nervo óptico $(\mathrm{Pu}-$ gliese et al. 2006).

Como se trata de um aparelho designado para uso em humanos, adaptações devem ser feitas para utilizá-lo na Medicina Veterinária. Para cada espécie são necessárias alterações específicas para a obtenção de imagens reprodutíveis e de alta resolução. Em cães e gatos, a presença de tapetum lucidum requer ajustes na iluminação para obtenção do corte óptico adequado. 0 uso de fármacos anestésicos pode promover rotação do bulbo ocular, sendo necessário o uso de pinças atraumáticas ou blefarostato, com intuito de manter o olho posicionado paralelamente ao equipamento (McLellan \& Rasmussen 2012).

Hernandez-Merino et al. (2011) padronizaram a técnica em cães, avaliando a retina e o nervo óptico. Cães Beagle isentos de oftalmopatias foram submetidos à OCT com o objetivo de analisar a espessura da retina e das camadas de fibras nervosas, fotorreceptores e nuclear externa. Os autores observaram que a espessura retiniana nas camadas de fotorreceptores e fibras nervosas era maior na área tapetal quando comparada à não tapetal, e que não houve diferença na camada nuclear externa, nessas duas áreas.

A OCT permite estratificar estruturalmente as camadas retinianas. No cão e no gato a camada de fibras nervosas, o EPR e a camada coriocapilar têm alta refletividade na área tapetal. $\mathrm{Na}$ área não tapetal, a região correspondente ao EPR e ao complexo coriocapilar apresenta hiporrefletividade. A camada plexiforme interna e a externa são mais hiper-refletivas que as camadas nucleares interna e externa. A camada de fotorreceptores apresenta baixa refletividade. Vasos retinianos são identificados pelo aumento da refletividade da área vascular e pela diminuição da refletividade de EPR e camada coriocapilar. Vasos coroidianos apresentam espaços ópticos vazios. Animais com lesão coriorretiniana, com hiper-reflexia e pigmento em razão de processo cicatricial, apresentam diminuição da espessura retiniana neurossensorial nas áreas acometidas, com alteração estrutural, além de hiper-refletividade das camadas EPR e coriocapilar em decorrência da pigmentação cicatricial (Pugliese et al. 2006).

A principal diferença na obtenção das imagens do homem e do cão é o posicionamento da cabeça, resultando em desafio para conseguir imagens de qualidade, principalmente em cães dolicocefálicos. Tal constatação reforça a necessidade de anestesiar os animais (Hernandez-Merino et al. 2011). No entanto Grahn et al. (2008), em estudo realizado em cães da raça Coton de Tulear, com intuito de avaliar descolamento retiniano seroso multifocal utilizaram OCT, obtendo imagens de boa qualidade, sem o emprego de sedativos ou anestésicos, pois os animais eram extremamente calmos e permitiram a realização dos exames.

As retinopatias podem ser classificadas em hereditárias ou adquiridas. As hereditárias podem ser classificadas em distrofias, displasias, tais como a degeneração progressiva de cones e bastonetes em Poodle e Cocker Spaniel. As retinopatias adquiridas correspondem a lesões retinianas causadas por agentes infecciosos, vasculopatias, neoplasias, afecções imunomediadas, metabólicas, tóxicas ou por radiação (Narfström \& Petersen-Jones 2007, Ofri 2008). A OCT auxilia, em muito, a deteç̧ão de retinopatias variadas, além de permitir o acompanhamento detalhado do tratamento ou da progressão das afecções (Hernandez-Merino et al. 2011). 0 objetivo deste estudo foi avaliar por meio da OCT a retina de cães hígidos e cães portadores de atrofia progressiva da retina (APR) e descolamento retiniano (DR).

\section{MATERIAL E MÉTODOS}

Foram examinados nove cães com idade entre três e 15 anos, de diferentes raças (Quadro 1), atendidos no Serviço de Oftalmologia do Departamento de Cirurgia e submetidos ao exame de OCT no Laboratório de Investigação em Oftalmologia Comparada, Faculdade de Medicina Veterinária e Zootecnia da Universidade de São Paulo.

Previamente foram realizados exames oftalmológicos tanto 
Quadro 1. Dados dos cães examinados por tomografia de coerência óptica incluídos no estudo

\begin{tabular}{lccccc}
\hline Cão & Raça & Sexo & $\begin{array}{c}\text { Idade } \\
\text { (anos) }\end{array}$ & Diagnóstico & $\begin{array}{c}\text { Espessura retinia- } \\
\text { na total }(\mu \mathrm{m})\end{array}$ \\
\hline 1 & Poodle & $\mathrm{F}$ & 9 & Hígido & 218 \\
2 & Lhasa Apso & $\mathrm{M}$ & 4 & Hígido & 219 \\
3 & Cocker Spaniel & $\mathrm{F}$ & 10 & Hígido & 221 \\
& Inglês & & & & \\
4 & SRD & $\mathrm{F}$ & 15 & Hígido & 230 \\
5 & Pinscher & $\mathrm{F}$ & 4 & Hígido & 236 \\
6 & Golden & $\mathrm{M}$ & 3 & DR $^{\mathrm{a}}$ & \\
7 & Retriever & & & & \\
8 & SRD & $\mathrm{F}$ & 6 & DR $^{2}$ & \\
9 & SRD & $\mathrm{M}$ & 6 & APR $^{\mathrm{b}}$ & \\
& SRD & $\mathrm{F}$ & 10 & APR $^{2}$ &
\end{tabular}

$\overline{{ }^{\mathrm{a}} \mathrm{DR}=\text { descolamento de retina; }}{ }^{\mathrm{b}} \mathrm{APR}=$ atrofia progressiva da retina.

nos animais sadios quanto nos portadores de afecções. Para a OCT os cães foram submetidos à sedação utilizando-se, por via subcutânea, associação de xilazina $2 \%(0,3 \mathrm{mg} / \mathrm{kg})$ e butorfanol $10 \mathrm{mg} / \mathrm{ml}(0,2 \mathrm{mg} / \mathrm{kg})$ ou associação de acepromazina $1 \%$ $(0,03 \mathrm{mg} / \mathrm{kg})$, meperidina $50 \mathrm{mg} / \mathrm{ml}(3,0 \mathrm{mg} / \mathrm{kg})$ e quetamina

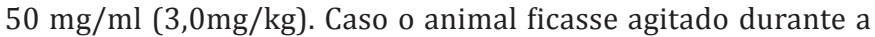
realização do exame, eram administrados, por via intravenosa, $2 \mathrm{mg} / \mathrm{kg}$ de propofol $10 \mathrm{mg} / \mathrm{ml}$, para evitar rotação do bulbo ocular. Os animais tiveram as pupilas dilatadas com colírio de tropicamida 1\% (Ciclomidrin, Latinofarma, São Paulo, Brasil). Dois casos com suspeita de atrofia retiniana foram submetidos ao exame de eletrorretinografia (ERG) (2000 Veris System, ElectroDiagnostic Imaging Inc., CA, USA), utilizando protocolo de cinco respostas de acordo com as diretrizes da International Society for Clinical Electrophysiology of Vision (ISCEV) (Marmor et al. 2009).

A escolha por contenção manual ou farmacológica por sedação ou anestesia dependeu do temperamento do paciente, pois este devia permanecer imóvel durante todo o procedimento para a obtenção de imagens de boa qualidade.

Após dilatação pupilar e posicionamento adequado do paciente, foram utilizadas, em alguns animais, pinças atraumáticas e blefarostato após instilação de anestésico tópico - cloridrato de proximetacaína $5 \mathrm{mg} / \mathrm{ml}$ (Anestalcon, Alcon, São Paulo, Brasil) com intuito de estabilizar o olho, evitando artefatos na imagem em razão da movimentação palpebral e ocular. Os animais foram posicionados em mesa pantográfica sendo que, para a execução do exame, a cabeça foi posicionada sobre o suporte do equipamento (Fig.1).

Promoveu-se o alinhamento do aparelho de tomografia de coerência óptica Spectralis SD-OCT (Spectral Domain Optical Coherence Tomography - Heidelberg Engineering Spectralis OCT; Heidelberg Engineering, CA, USA) e de seu foco para obtenção de imagem nítida da região a ser estudada no monitor. Cortes radiais ("radial lines") e cortes paralelos ("rasterlines") foram usados com intuito de escanear a retina.

Os olhos foram lubrificados constantemente com solução fisiológica $0,9 \%$ e realizada aplicação frequente de lubrificante artificial (Systane, Alcon, São Paulo, Brasil) a fim de evitar diminuição na qualidade das imagens decorrente de ressecamento corneal.

As imagens tomográficas que representavam a refletividade da retina foram mostradas bi ou tridimensionalmente em tempo real em uma falsa escala de cores ou em branco e preto.

A presença do tapetum lucidum nos cães requereu ajustes no aparelho de OCT, como a escolha no parâmetro de iluminação e foco.

Todos os experimentos receberam a aprovação da comissão de ética da Faculdade de Medicina Veterinária e Zootecnia da Universidade de São Paulo, protocolo número 2972/2013.

\section{RESULTADOS}

Foram obtidas imagens da retina, em perfil, espessura e em três dimensões nos cães avaliados pela OCT. Em razão da excelente qualidade de imagem, foi possível distinguir facilmente as camadas retinianas (Fig.2). No Quadro 1 estão descritos os valores mensurados da retina (neurossensorial e EPR) obtidos nos cães hígidos, sendo a área tapetal a principal área avaliada.

O DR foi diagnosticado em dois casos pela fundoscopia direta e pelo exame de OCT (Fig.3). Em um caso o DR foi mais evidente na área não tapetal (Fig.4). Em ambos os animais o DR cursou com déficit visual avaliado pela ausência dos reflexos pupilar direto, consensual e de ameaça. Notou-se uma área de hiporrefletividade entre as camadas membrana limitante/segmento interno e externo e o EPR, correspondendo ao descolamento seroso. Obser-

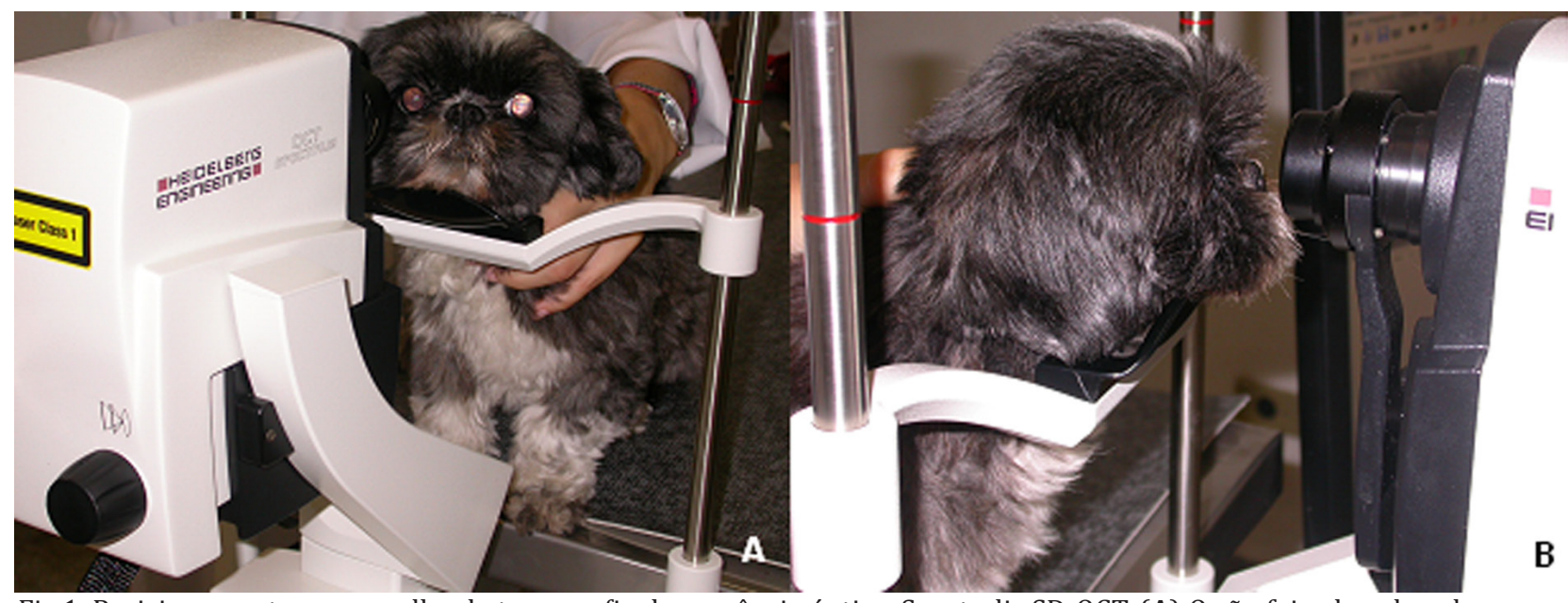

Fig.1. Posicionamento no aparelho de tomografia de coerência óptica, Spectralis SD-OCT. (A) 0 cão foi colocado sobre mesa pantográfica e o focinho posicionado no suporte do equipamento. (B) 0 olho é voltado diretamente para a lente do aparelho para obtenção da imagem. 


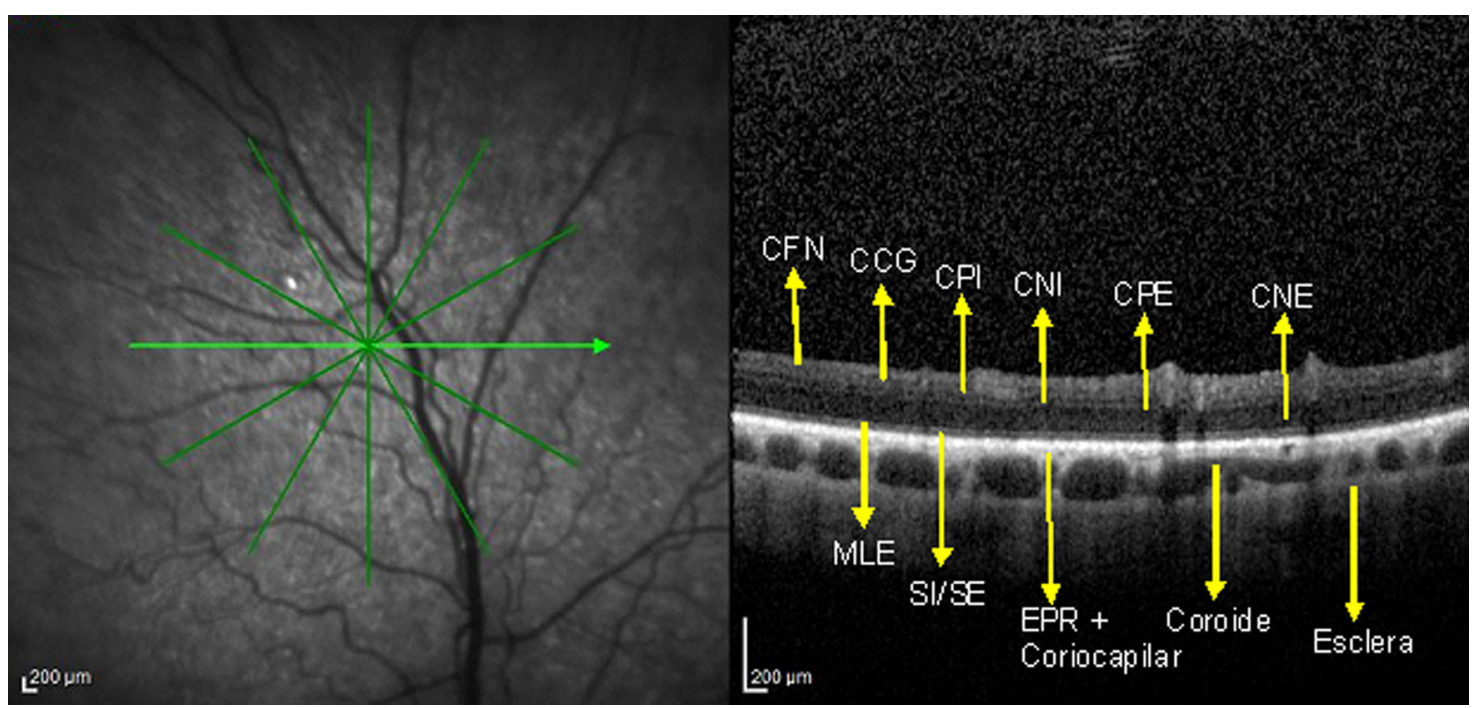

Fig.2. Imagem de OCT utilizando cortes radiais da retina normal de cão Poodle de 9 anos. As camadas retinianas identificadas são: camada de fibras nervosas (CFN), camada de células ganglionares (CCG), camada plexiforme interna (CPI), camada nuclear interna (CNI), camada plexiforme externa (CPE), camada nuclear externa (CNE), membrana limitante externa (MLE), linha de junção do segmento interno e externo dos fotorreceptores (SI/SE), camada do epitélio pigmentado da retina (EPR).

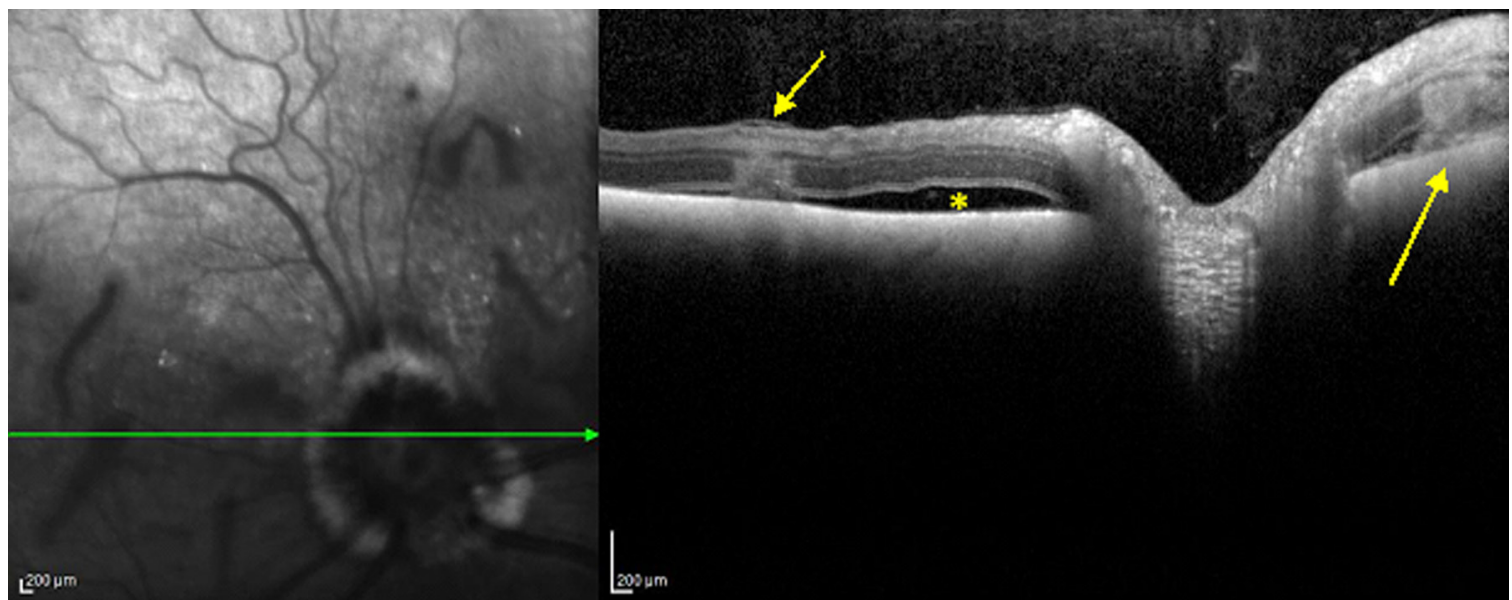

Fig.3. Imagem de OCT (esquerda) de descolamento de retina (DR) da retina peripapilar de um Golden Retriever, macho de 3 anos. Observa-se espaço hiporrefletivo entre a camada neurossensorial e EPR, característico de descolamento seroso (asterisco). Próximo ao DR e à papila do nervo óptico nota-se uma área hiper-refletiva, homogênea, bem delimitada, promovendo sombreamento posterior, sugestivo de processo hemorrágico (setas).

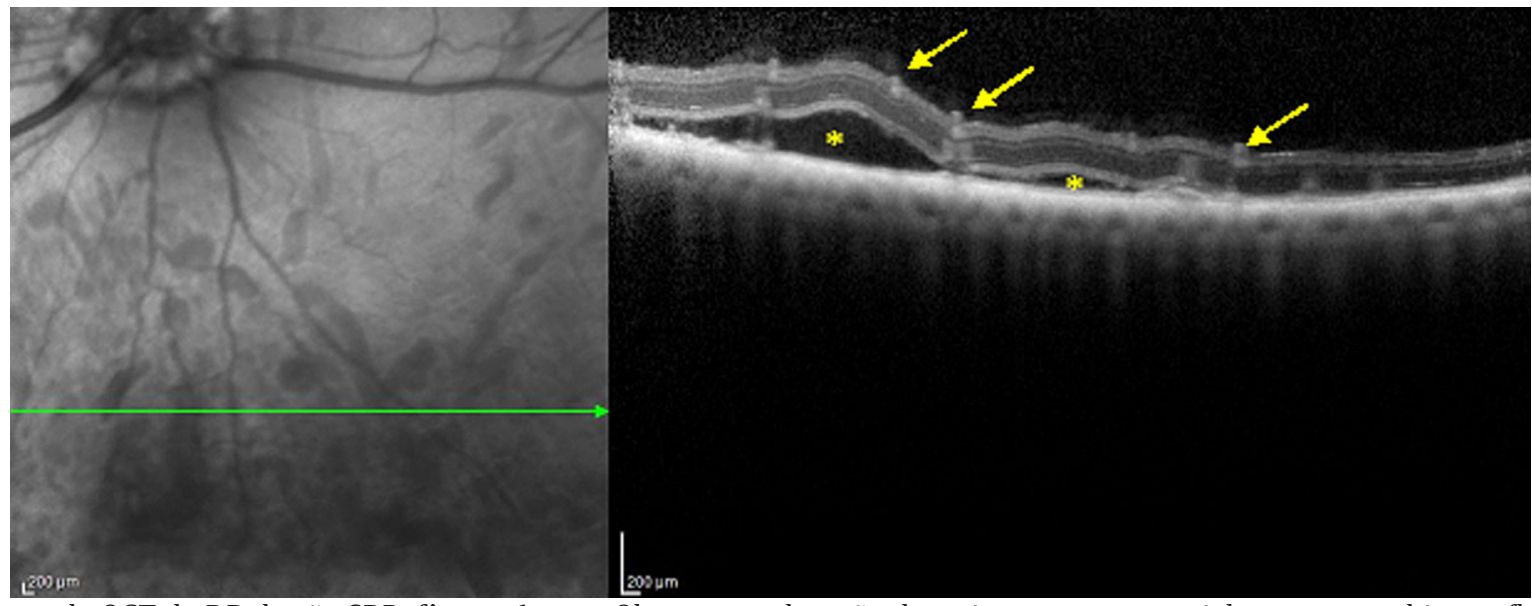

Fig.4. Imagem de OCT de DR de cão SRD, fêmea, 6 anos. Observa-se elevação da retina neurossensorial com espaço hiporrefletivo sub-retiniano correspondente a descolamento seroso (asteriscos). Presença de múltiplas lesões hiper-refletivas na camada externa, sugestivas de hemorragia intrarretiniana (setas). 


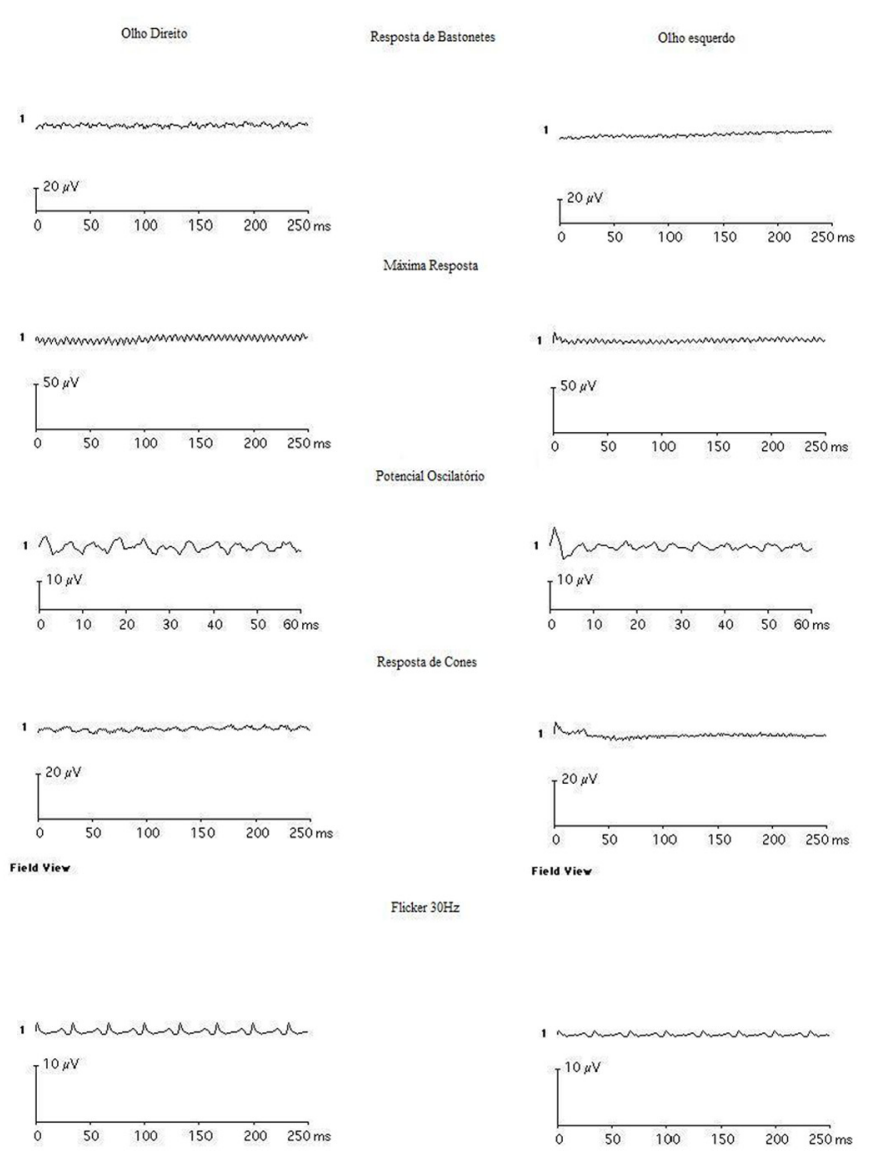

Fig.5. Eletrorretinograma de campo total com cinco respostas (Resposta de bastonetes, Máxima resposta, Potencial Oscilatório, Resposta de cones e Flicker a $30 \mathrm{~Hz}$ ), utilizando eletrorretinógrafo Veris 2000, realizado em ambos os olhos de um cão SRD, macho, seis anos, portador de APR. Observar ausência de respostas eletrorretinográficas. varam-se alguns pontos de hiper-refletividade intrarretiniana, compatíveis com processo exsudativo e/ou hemorrágico. Ambos os cães permitiram o exame sem protocolo anestésico.

Em dois cães com diagnóstico de atrofia retiniana pela oftalmoscopia direta observou-se hiper-reflexia da área tapetal e atenuação vascular significativa. A ERG mostrou ausência de respostas ao estímulo luminoso (Fig.5). Submetidos a OCT, notou-se atrofia significativa nas camadas da retina. A área tapetal foi avaliada por cortes paralelos (rasterlines) e, em ambos os casos, observou-se que a área da retina próxima ao nervo óptico apresentou maior espessura em comparação com a periferia da retina (Fig. 6 e 7). Dependendo do corte avaliado foi possível notar completa perda da estratificação, ou seja, afinamento de todas as camadas da retina neurossensorial.

\section{DISCUSSÃO}

A finalidade deste estudo foi estudar a viabilidade do uso da OCT em duas importantes retinopatias em cães, o descolamento de retina e a atrofia progressiva da retina.

A OCT permite a realização de cortes ópticos transversais da retina, gerando imagens de alta resolução, que se aproximam da resolução obtida na microscopia óptica (Malerbi et al. 2010). 0 emprego do corte óptico permitiu distinguir limites entre as camadas intrarretinianas e a interface vitreorretiniana (Guia et al. 2010). Cortes radiais ("radial lines") e cortes paralelos ("rasterlines") também foram empregados com intuito de escanear a retina, dependendo do tamanho da região examinada.

A contenção manual e/ou farmacológica fez-se necessária para a execução do exame, pois a qualidade do exame dependia do perfeito posicionamento do paciente. Vários são os protocolos anestésicos encontrados na Medicina Ve-

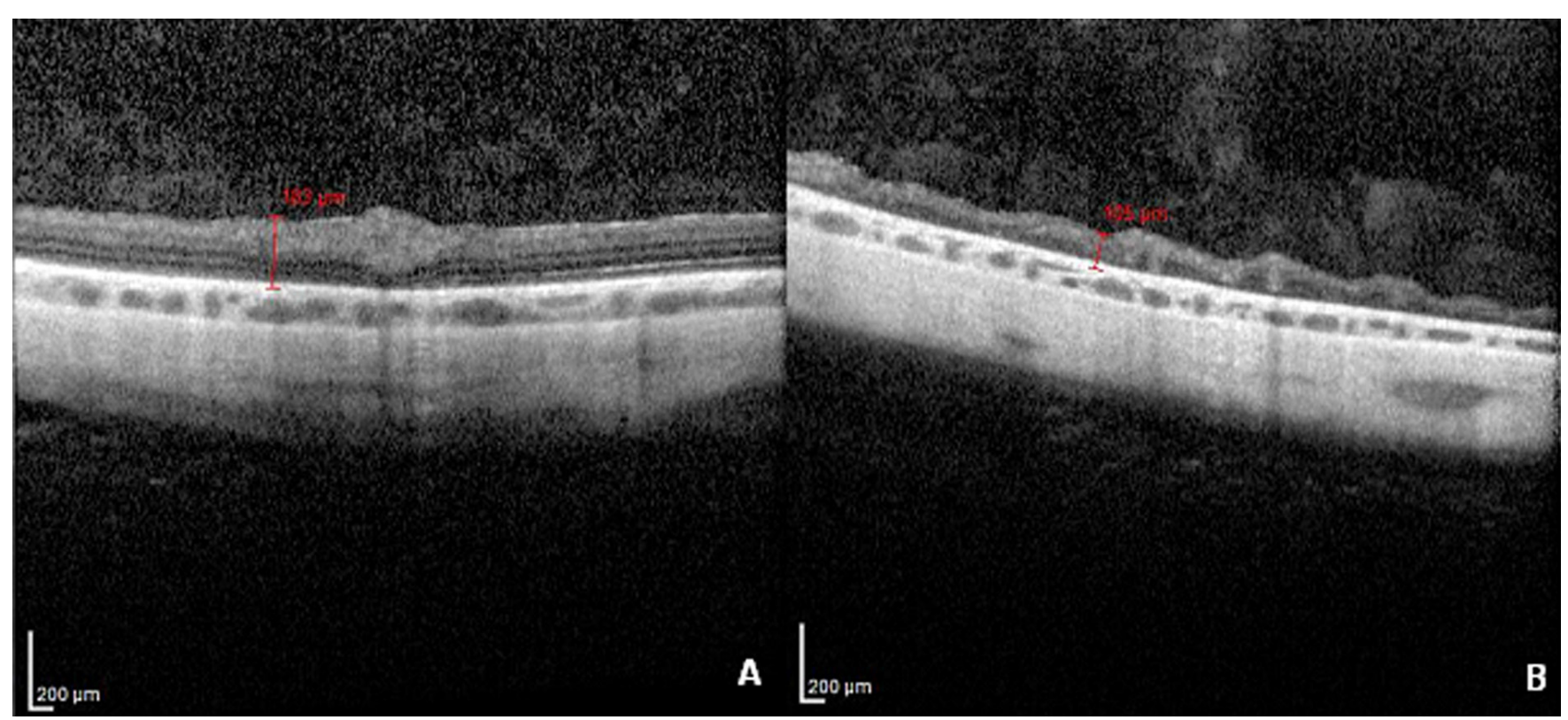

Fig.6. Imagem de OCT da área tapetal de cão SRD, macho, seis anos submetido ao ERG de Campo total. Observa-se afinamento da retina neurossensorial. (A) A região avaliada próxima ao nervo óptico com espessura de $183 \mu \mathrm{m}$, enquanto que, em (B) nota-se diminuição e desorganização importante das camadas da retina em região periférica, com espessura de $105 \mu$ m e diversas elevações na camada de fibras nervosas. 


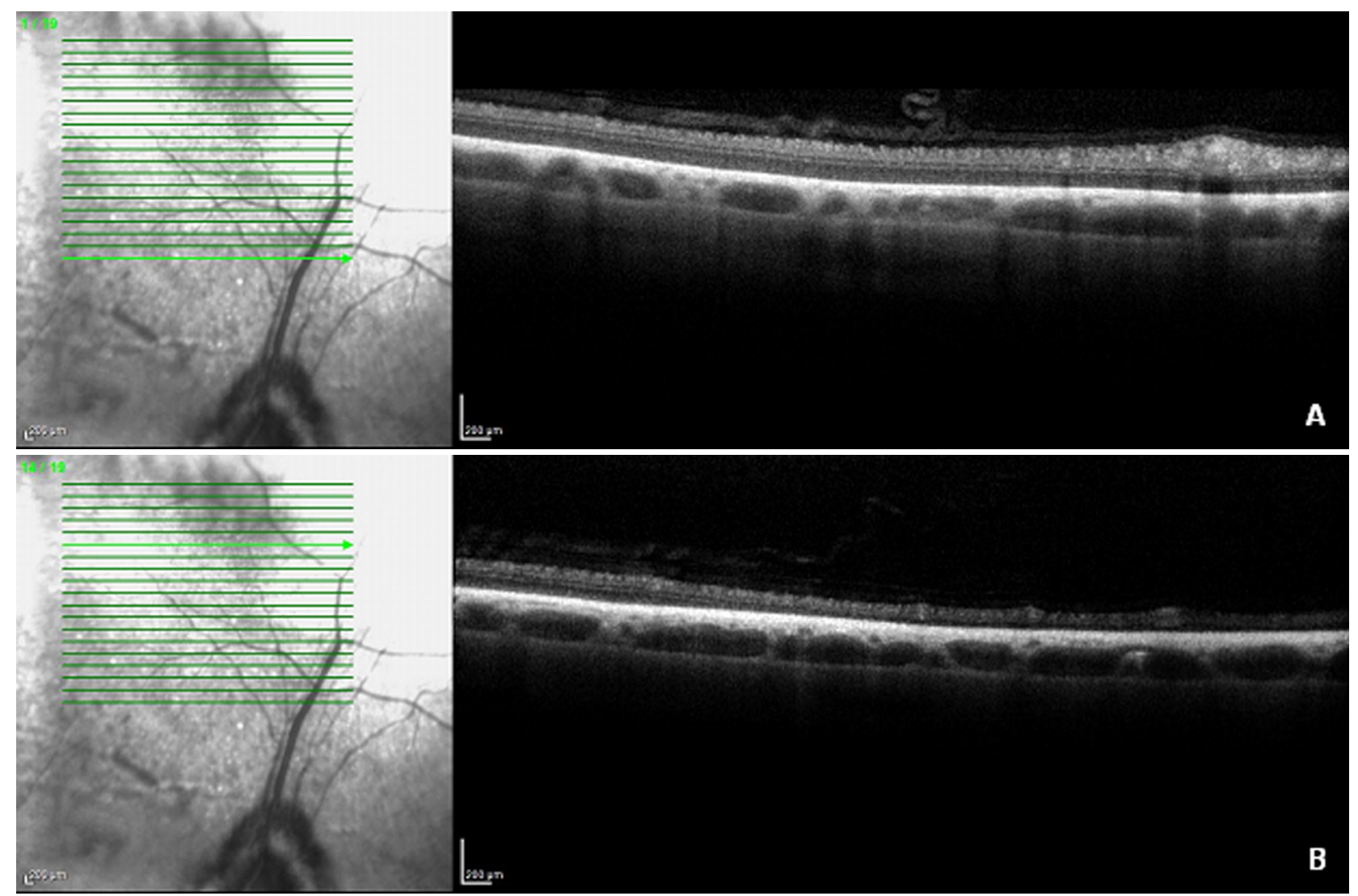

Fig.7. Imagem de OCT com cortes paralelos, cão SRD, fêmea de 10 anos. (A) Os cortes na região peripapilar (região central) apresentam retina neurossensorial com redução difusa da camada externa e estratificação da camada interna preservada. (B) Os cortes da região periférica demonstram afinamento mais acentuado e desorganização de arquitetura interna, sem identificação das estratificações.

terinária nas diversas espécies. Como o emprego de fármacos anestésicos ou sedativos não interfere nos resultados obtidos na OCT, avaliou-se a necessidade de empregá-los caso a caso. A maioria dos animais foi sedada com xilazina associada ao butorfanol, que facilitou a obtenção de imagens de boa qualidade. 0 mesmo também aconteceu com os animais nos quais foi utilizado o propofol. Dois cães foram apenas contidos manualmente, em razão de seu temperamento dócil. A contenção manual foi descrita em um estudo com 30 cães da raça Coton de Tulear submetidos a exames oftalmológicos, incluindo OCT, que não necessitaram de protocolo anestésico, por serem cães extremamente calmos (Grahn et al. 2008).

A dilatação pupilar foi adequadamente obtida com a instilação prévia de midriático e também instituída em outros estudos, bem como a realização do exame em sala semiescurecida (Rosolen et al. 2012). Apesar da OCT também ser realizada em pupilas não dilatadas a dilatação pupilar facilita o exame (Malerbi et al. 2010).

A movimentação ocular dificulta, em muito, a aquisição de boas imagens para sua interpretação. Alguns autores utilizam clips atraumáticos (Rosolen et al. 2012) ou bloqueadores neuromusculares para suprimir a movimentação ocular (Hernandez-Merino et al. 2011). Em alguns casos de nosso estudo foram utilizadas pinças atraumáticas e/ou blefarostato, com o olho previamente anestesiado com colírio anestésico, com intuito de estabilizá-lo, possibilitando, assim, a obtenção de imagens de boa qualidade.
Em relação à espessura das camadas da retina, os valores encontrados para a área tapetal foram de 218 a $236 \mu \mathrm{m}$ nos cães hígidos. Hernandez-Merino et al. (2011) obtiveram valor médio de $198,7 \mu \mathrm{m}$ para a mesma região. Contudo, Panzan et al. (2004) determinaram valores semelhantes na área tapetal, analisando a regiões nasal e temporal, sendo $183,7 \mu \mathrm{m}$ e $217,8 \mu \mathrm{m}$, respectivamente.

Lesões observadas no segmento posterior do olho pela oftalmoscopia e pela retinografia podem ser melhor caracterizadas pela OCT, que identifica claramente quais camadas da retina estão envolvidas (McLellan \& Rasmussen 2012). Assim, a OCT tem sido aplicada com êxito para detectar alterações estruturais na retina em humanos, tais como mensuração da camada de fibras nervosas para monitorização em pacientes com glaucoma e mensuração da camada de fotorreceptores na retinose pigmentária (Gekeler et al. 2007); esta caracterizada por alteração genética que afeta a camada de fotorreceptores e o EPR. 0 diagnóstico é baseado nos achados fundoscópicos e na avaliação do ERG (Witkin et al. 2006). Em cães, a APR assemelha-se à retinose pigmentária em humanos, pelo histórico e pela característica clínica. Panzan et al. (2004) realizaram OCT em cães portadores de displasia dos fotorreceptores, uma variação da APR, e constataram diminuição de espessura da area centralis da retina em relação à retina de cães hígidos. Este dado corrobora os achados deste estudo, no entanto a diminuição de espessura ocorreu de maneira evidente na região periférica nos dois casos avaliados. 
Descolamento de retina, também chamado de separação da retina, ocorre entre o EPR e a retina neurossensorial ou neurorretina (McLellan 2008). Em humanos com DR, a avaliação pela OCT pode ser útil por revelar fluidos intra ou sub-retinianos, não evidenciados por meio de outras técnicas, indicando atividade da lesão (Zanotele et al. 2010). Neste estudo foi possível avaliar o DR e a presença de interfaces hiper-refletivas que corresponderam a exsudatos e uma área hiporrefletiva adjacente ao DR compatível com presença de fluidos. Tais alterações cursaram com déficit visual em ambos os casos avaliados.

\section{CONCLUSÃO}

A tomografia de coerência óptica proporcionou identificação das camadas retinianas em cães hígidos e permitiu comparação com as características retinianas em cães portadores de DR e APR. A OCT é uma ferramenta útil e inovadora na avaliação microscópica da retina in vivo, permitindo um diagnóstico acurado das retinopatias em cães, além de permitir o avanço nas pesquisas em retinopatias.

Agradecimentos.- À FAPESP pelo auxílio financeiro a projeto de pesquisa (2011/24039-8).

\section{REFERÊNCIAS}

Allemann N., Ferraz C.A., Chalita M.E., Baïkoff G. \& Farah M.E. 2010. Córnea e segmento anterior, p.387-391. In: Farah M.E. (Ed.), Tomografia de Coerência Óptica: OCT. $2^{\underline{a}}$ ed. Guanabara Koogan, Rio de Janeiro.

Barnett K.C., Sansom J. \& Heinrich C. 2002. Retinal detachment, p.155-179. In: Barnett K.C., Sansom J. \& Heinrich C. (Eds), Canine Ophthalmology: an atlas and text. W.B. Saunders, London.

Bordon A.F., Oshima A., Guia T.A., Calucci D., Sallum J.M. \& Farah M.E. 2008. Ocular coherence tomography in age-related macular degeneration patients treated with photodynamic therapy with verteporforin. Arq. Bras. Oftalmol. 71(6):799-804.

Fisher M.D., Huber G., Beck S.C., Tanimoto N., Muehfriedel R., Fahl E., Grimm C., Wenzel A., Reme C.E., Van de Pavert S., Wijnholds J., Pacal M., Bremmer R. \& Seeliger M.W. 2009. Noninvasive, in vivo assessment of mouse retinal structure using optical coherence tomography. Public Library of Science 4(10):1-7.

Gekeler F., Gmeiner H., Volker M., Sachs H., Messias A., Eule C.,BartzSchmidt K.U., Zrenner E. \& Shinoda K. 2007. Assessment of the posterior segment of the cat eye by optical coherence tomography (OCT). Vet. Ophthalmol. 10(3):173-178.

Gouveia E.B., Morales M.S.A., Allemann N., Matte G., Berezovsky A. \& Sallum J.M.F. 2006. Aspectos da tomografia de coerência óptica na doença de Stargardt: relato de caso. Arq. Bras. Oftalmol. 69(4):589-592.

Grahn B.H., Sandmeyer L.L. \& Breaux C. 2008. Retinopathy of coton de tulear dogs: clinical manifestations, electroretinographic, ultrasonographic, fluorescein and indocyanine green angiographic, and optical coherence tomographic findings. Vet. Ophthalmol. 11(4):242-249.

Guedes V. \& Yamane R. 2003. Semiologia ocular, p.225-236. In: Yamane R. (Ed.), Tomografia de Coerência Óptica. $2^{\underline{a}}$ ed. Cultura Médica, Rio de Janeiro.

Guia T., Mello Filho P.A.A., Endo M.Y. \& Farah M.E. 2010. Programas aplicativos, p.13-32. In: Farah M.E. (Ed.), Tomografia de Coerência Óptica: OCT. $2^{\underline{a}}$ ed. Cultura Médica, Guanabara Koogan, Rio de Janeiro.
Hernandez-Merino E., Kecova H., Jacobson S.J., Hamouche K.N., Nzokwe R.N. \& Grozdanic S.D. 2011. Spectral domain optical coherence tomography (SD-OCT) assessment of healthy female canine retina and optic nerve. Vet. Ophthalmol. 14(6):400-405.

Huang D., Swanson E.A., Lin C.P., Schuman J.S., Stinson W.G. \& Chang W. 1991. Optical Coherence Tomography. Science 254(5035):1178-1181.

Kloizman T.P., Pakter H.M., Schuman J.S., Szwartz J.C. \& Hee M.R. 1998. Ophthalmic diagnosis using optical coherence tomography. Ophthalmol. Clin. North Am. 11:465-486.

Malerbi F.K., Andrade R.E.A. \& Farah M.E. 2010. OCT no Diagnóstico por Imagem, p.1-8. In: Farah M.E. (Ed.), Tomografia de Coerência Óptica: OCT. $2^{a}$ ed. Cultura Médica, Guanabara Koogan, Rio de Janeiro.

Marmor M.F., Fulton A.B., Holder G.E., Miyake Y., Brigell M. \& Bach M. 2009. ISCEV standard for full-field clinical electroretinography (2008 update), Doc. Ophthalmol. 118:69-77.

McLellan G. 2008. The Canine Fundus, p.227-246. In: Petersen-Jones S. \& Crispin S. (Eds), Small Animal Ophthalmology. 2nd ed. BSAVA, England.

McLellan G.J. \& Rasmussen C.A. 2012. Optical coherence tomography for the evaluation of retinal and optic nerve morphology in animal subjects: practical considerations. Vet. Ophthalmol. 15:13-28.

Narfström K. \& Petersen-Jones S. 2007. Disease of the canine ocular fundus, p.944-1025. In: Gelatt K.N. (Ed.), Veterinary Ophthalmology. 4th ed. Blackwell Publishing, Iowa.

Ofri R. 2008. Lens, p.258-276. In: Maggs D.J., Miller P.E. \& Ofri R.I. (Eds), Slatter's Fundamentals of Veterinary Ophthalmology. 4th ed. Saunders Elsevier, St Louis.

Panzan C.Q., Guven D., Weiland J.D., Lakhanpal R.R., Javaheri M., Júnior J.E. \& Humayun M.S. 2004. Retinal thickness in normal and RCD1 dogs using optical coherence tomography. Ophthal. Surg. Lasers Imaging 35(6):485-493.

Pugliese A., Trombetta C.J., Bonanno G., Freno M.C., Nasso S. \& Di Pietro S. 2006. OCT in the dog and cat: morpho-structural patterns and clinical features of the fundus. Vet. Res. Comun. 30:313-315.

Rosolen S.G., Rivière M.L.K., Lavillegrand S., Gautier B., Picaud S. \& Legargasson J.F. 2012. Use of combined slit-lamp SD-OCT to obtain anterior and posterior segment images in selected animal species. Vet. Ophthalmol. 15:101-115.

Saraiva V.D.S., Aggio F.B., Júnior M.N.B. \& Farah M.E. 2010. Correlação anatômica, p.45-8. In: Farah M.E. (Ed.), Tomografia de Coerência Óptica: OCT. $2^{a}$ ed. Cultura Médica. Guanabara Koogan, Rio de Janeiro.

Schuman J.S. 1997. Imaging in glaucoma, p.95-130. In: Schuman J.S. (Ed.), Optical Coherence Tomography for Imaging and Quantitation of Nerve Fiber Laber Layer Thickness. Slack, Thorofare.

Song S.E., Aggio F.B. \& Farah M.E. 2010. Distrofias maculares, p.285-288. In: Farah M.E. (Ed.), Tomografia de Coerência Óptica: OCT. $2^{a}$ ed. Cultura Médica, Guanabara Koogan, Rio de Janeiro.

Thomas D. \& Duguid G. 2004. Optical coherence tomography: a review of the principles and contemporary uses in retinal investigation. Eye 18:561-570.

Zanotele M., Aggio F.B., Duprat J.P.N. \& Farah M.E. 2010.Descolamento do epitélio pigmentado da retina, p.101-106. In: Farah M.E. (Ed.), Tomografia de Coerência Óptica: OCT. $2^{\mathrm{a}}$ ed. Cultura Médica, Guanabara Koogan, Rio de Janeiro.

Witkin A.J., Ko T.H., Fujimoto J.G., Chan A., Drexler W., Schuman J.S., Reichel E. \& Duker J.S. 2006. Ultra-high resolution optical coherence tomography assessment of photoreceptores in retinitis pigmentosa and related disease. Am. J. Ophthalmol. 142(6):945-952. 